demonstrated a tighter transition from the second to third segment of the duodenum, with no mucosal abnormalities, consistent with extraluminal compression. Computed tomography with intravenous contrast was ordered which revealed reduced aortomesenteric angle and distance; findings which established the diagnosis of Superior Mesenteric Artery Syndrome.

Conclusion Most cases of Superior Mesenteric Artery Syndrome are presentations of chronic duodenal obstruction. Acute forms can occur in the face of trauma. Our patient is an interesting case, who continued to thrive with height and weight proportional, and was atraumatic. On review in outpatient facility recurrence of the epigastric pain was found to coincide with a recent growth spurt. A potential new association for exacerbation of asymptomatic chronic duodenal obstruction due to Superior Mesenteric Artery Syndrome.

\section{GP58 CASE REPORT OF HUNTER SYNDROME IN MONGOLIANS}

'Zolzaya Doljoo*, ${ }^{2}$ Sarantuya Jav, ${ }^{2}$ Purevdorj Ichinkhorloo, ${ }^{1}$ Batchimeg Batbaatar, 'Oyungerel Ganjuur, ${ }^{2}$ Munkhbat Batmunkh, ${ }^{1}$ Munkhtuvshin Namid, ${ }^{1}$ Munkhtuya Tumurkhuu. 'Institute of Medical Sciences, Ulaanbaatar, Mongolia; ${ }^{2}$ Mongolian National University of Medical Sciences, Ulaanbaatar, Mongolia

10.1136/archdischild-2019-epa.124

Background Mucopolysaccaridosis II (MPSII), Hunter syndrome, (OMIM\# 309900) is a metabolic genetic disorder caused by deficiency of iduronate-2-sulfatase (IDS) enzyme necessary for step-wise degradation of glycosaminoglycan (GAG) in a cell. Point mutations and small deletions of exon 9 of IDS gene account for 50\% of Hunter syndrome cases. Accumulation of GAG virtually in all cells causes MPS that is characterized by mental, growth progressive retardation, multiple system dysfunction and death usually occurs in first and second decade of life. MPS cases are diagnosed by only clinical symptoms in our country and detection of mutation, identification of defective enzymes, and carrier detection in the patient's family is still missing. Purpose of this study was to diagnose MPS cases by molecular genetic analysis and detect carriers in case if mutation detected.

Methods Iduronate-2 sulfatase enzyme activity was checked by tandem mass spectrometer. IDS gene mutation was analyzed in Hunter syndrome patients by direct sequencing. We used Multiplex Ligation-dependent Probe Amplification (MLPA) assay to detect $\times$ chromosome deletion in case if we could not amplify IDS gene.

Results Totally we investigated four patients with MPS, and familial members of a case 3, who had a mutation in IDS gene. Proband-E showed clinical symptoms of Hurler syndrome, but Hunter syndrome was suspected by genealogical study, also enzyme deficiency of iduronate 2 sulfatase was used to differentiate his diagnosis. We could not amplify exons of IDS gene in this patient sample, suggesting that he might have a deletion in $\times$ chromosome, and also we could not identify deletion covering Xq28 using SALSA MPLA probemix P095-A3 Aneuploidy. Proband-A is from inbred parents, in whom we suggest to have autosomal recessive mutant alleles. Proband-T showed clinical symptoms of Hunter syndrome and pedigree analysis and enzyme deficiency analysis confirmed it. Mutation screening was done in exon $2,3,8$ and 9 of IDS gene where most mutations reported in Hunter syndrome patients and a R468W mutation leading to a replacement of arginine 468 to tryptophan was detected in the patient. His mother carries mutant allele, as well as his three sisters. Proband I expresses clinical symptoms of Hunter syndrome and iduronate 2 sulfatase enzyme deficiency detected in blood samples which is confirmatory for Hunter syndrome.

Conclusion This is a first genetic analysis of MPS cases in Mongolia. We detected R468W mutation in the patient with severe phenotype of Hunter syndrome. We also confirmed four female carriers in this family.

\section{GP59 A RARE CAUSE OF 'MITOCHONDRIAL DISORDER': COCKAYNE SYNDROME}

${ }^{1}$ Arie Fisher* ${ }^{*}{ }^{1}$ Muhammad Asghar, ${ }^{2}$ Stephanie Ryan, ${ }^{3}$ Bryan Lynch, ${ }^{4}$ Andrew Green, ${ }^{1}$ Ina Knerr. 'National Centre for Inherited Metabolic Disorders, Temple Street Children's University Hospital, Dublin, Ireland; 'Department of Radiology, Temple Street Children's University Hospital, Dublin, Ireland; ${ }^{3}$ Department of Neurology, Temple Street Children's University Hospital, Dublin, Ireland; ${ }^{4}$ Department of Clinical Genetics, Temple Street Children's University Hospital, Dublin, Ireland

\subsection{6/archdischild-2019-epa.125}

A 16 month old toddler presented with global developmental delay, truncal hypotonia and microcephaly. Her background history was significant for bilateral developmental dysplasia of the hip, recurrent upper respiratory tract infections, conductive hearing loss and a scalp haemangioma. Development had been apparently normal up until 6 months of age.

Over the course of her follow-up she developed signs of cerebellar dysfunction including intention tremor and broad based ataxia, chronic feeding difficulties, pigmentary retinopathy and skin photosensitivity. MRI brain at age 2.5 years showed delayed myelination and cerebellar atrophy. A skeletal muscle biopsy revealed reduced activities for the respiratory chain complexes I, II and IV, in particular, in keeping with a mitochondrial condition. After extensive genetic investigations failed to identify the underlying aetiology, an exome sequencing study was carried out and revealed a mutation in the ERCC6 DNA repair gene, consistent with Cockayne syndrome (OMIM \# 133540). This is a rare autosomal recessive genetic condition which causes microcephaly, developmental delay and failure to thrive, along with sun sensitivity, tooth decay, bone abnormalities, hearing and vision loss and other symptoms. The patient has been managed with multidisciplinary care and symptomatic treatment, including skin protection, as well as nutritional and multivitamin supplements. She is wheelchair bound and non-verbal but otherwise medically well at age 7 yrs.

Many clinical signs seen in patients with Cockayne syndrome are also found in patients with a primary mitochondrial disease. Conversely, secondary dysfunctional mitochondria may also contribute to the phenotype of patients with Cockayne syndrome. Taken together, this case demonstrates the value of exome sequencing in diagnosing and differentiating rare disorders with diverse and overlapping phenotypes, including primary and secondary mitochondrial conditions, which are less likely to be diagnosed on clinical grounds and biochemical investigations alone. 